\title{
Cholinergic dysfunction in a mouse model of Alzheimer disease is reversed by an anti-A $\beta$ antibody
}

\author{
Kelly R. Bales, ${ }^{1,2}$ Eleni T. Tzavara, ${ }^{1}$ Su Wu, ${ }^{1}$ Mark R. Wade, ${ }^{1}$ Frank P. Bymaster, ${ }^{1}$ \\ Steven M. Paul, ${ }^{1}$ and George G. Nomikos ${ }^{1}$ \\ ${ }^{1}$ Neuroscience Discovery Research, Eli Lilly and Company, Indianapolis, Indiana, USA. ${ }^{2}$ Program of Medical Neurobiology, \\ Indiana University School of Medicine, Indianapolis, Indiana, USA.
}

\begin{abstract}
Disruption of cholinergic neurotransmission contributes to the memory impairment that characterizes Alzheimer disease (AD). Since the amyloid cascade hypothesis of $\mathrm{AD}$ pathogenesis postulates that amyloid $\beta$ (A $\beta$ ) peptide accumulation in critical brain regions also contributes to memory impairment, we assessed cholinergic function in transgenic mice where the human $A \beta$ peptide is overexpressed. We first measured hippocampal acetylcholine $(\mathrm{ACh})$ release in young, freely moving PDAPP mice, a well-characterized transgenic mouse model of $A D$, and found marked $A \beta$-dependent alterations in both basal and evoked $A C h$ release compared with WT controls. We also found that $A \beta$ could directly interact with the high-affinity choline transporter which may impair steady-state and on-demand ACh release. Treatment of PDAPP mice with the anti-A $\beta$ antibody m 266 rapidly and completely restored hippocampal ACh release and high-affinity choline uptake while greatly reducing impaired habituation learning that is characteristic of these mice. Thus, soluble "cholinotoxic" species of the $A \beta$ peptide can directly impair cholinergic neurotransmission in PDAPP mice leading to memory impairment in the absence of overt neurodegeneration. Treatment with certain anti-A $\beta$ antibodies may therefore rapidly reverse this cholinergic dysfunction and relieve memory deficits associated with early AD.
\end{abstract}

\section{Introduction}

The cholinergic neurotransmitter system in brain is critical for the processing of information related to cognitive function (1). The nearly complete destruction of cholinergic neurons located within the nucleus basalis of Meynert in Alzheimer disease (AD), has led many investigators to postulate that cholinergic dysfunction is a primary cause of the memory decline associated with AD (2-4). An alternative but not mutually exclusive hypothesis of AD pathogenesis, the "amyloid cascade hypothesis," postulates that memory deficits are caused by increased brain levels of both soluble and insoluble amyloid $\beta(A \beta)$ peptide(s), which are derived from the larger amyloid precursor protein (APP) by sequential proteolytic processing (5). Although no direct clinical evidence in support of this hypothesis is yet available, ample genetic evidence derived from mutations within the APP gene associated with familial early-onset forms of $\mathrm{AD}$ supports an important role for the $\mathrm{A} \beta$ peptide(s) in AD pathogenesis (6). In addition to the abundant deposits of $\mathrm{A} \beta$ in brain parenchyma of $\mathrm{AD}$ patients, there are also neuritic plaques and neurofibrillary tangles within the basal forebrain and neocortical cholinergic pathways $(3,4)$. Although deficits in several neurotransmitter systems have been observed in $\mathrm{AD}$ brain, basal forebrain cholinergic neurons appear to be exquisitely sensitive and susceptible to the disease process, and the majority of currently available therapies, which do not alter disease progression, target the cholinergic synapse in an attempt to increase synaptic levels of acetylcholine (ACh) in order to relieve the memory deficits associated with disease progression.

Nonstandard abbreviations used: $A \beta$, amyloid $\beta ; A C h$, acetylcholine; $A D$, Alzheimer disease; APP, amyloid precursor protein; ChT-1, choline transporter.

Conflict of interest: All authors are or have been employed by Eli Lilly and Company. Citation for this article: J. Clin. Invest. doi:10.1172/JCI27120.
Both soluble and insoluble forms of the $\mathrm{A} \beta$ peptide(s) have been shown to disrupt synaptic transmission and inhibit long-term potentiation in vivo as well as to cause memory impairment in transgenic mouse models of $\mathrm{AD}$, which overexpress mutations associated with familial forms of $\operatorname{AD}(7,8)$. Moreover several studies in humans have demonstrated significant correlations between cognitive impairment and the level of soluble (9-11) and certain deposited forms of $A \beta$ (12). Additionally, we have recently demonstrated that administration of the anti-A $\beta$ antibody $\mathrm{m} 266$, which binds with very high affinity to the mid-domain region of the soluble forms of $A \beta$, is able to rapidly reverse memory impairment in PDAPP mice following acute or subchronic administration without any measurable change in brain $A \beta$ burden (13).

To investigate whether the $A \beta$ peptide(s) may directly affect cholinergic function in the absence of overt neurodegeneration, we measured hippocampal ACh release using in vivo microdialysis in awake, freely moving transgenic mice that overexpress a mutation associated with familial AD (PDAPP mice). PDAPP mice represent a well-characterized animal model of AD-like plaque pathology with $A \beta$ and amyloid deposition occurring in an age- and brain region-dependent fashion (14). Although these mice have behavioral deficits, they do not develop neurodegeneration or frank loss of cholinergic neurons even as they age (15-17). Here we report an $\mathrm{A} \beta$-dependent disruption of hippocampal ACh release in PDAPP mice that was associated with impaired habituation learning. Kinetic analysis of high-affinity choline uptake into synaptosomes prepared from the hippocampus of PDAPP mice demonstrated a significant increase in $V_{\max }$ without any measurable effect on $K_{\mathrm{m}}$. We also observed a marked effect of $A \beta_{42}$ on high-affinity choline uptake in rat hippocampal synaptosomes or membranes prepared from a stable cell line expressing the human choline transporter (ChT-1). Additionally, we utilized coimmunoprecipitation fol- 

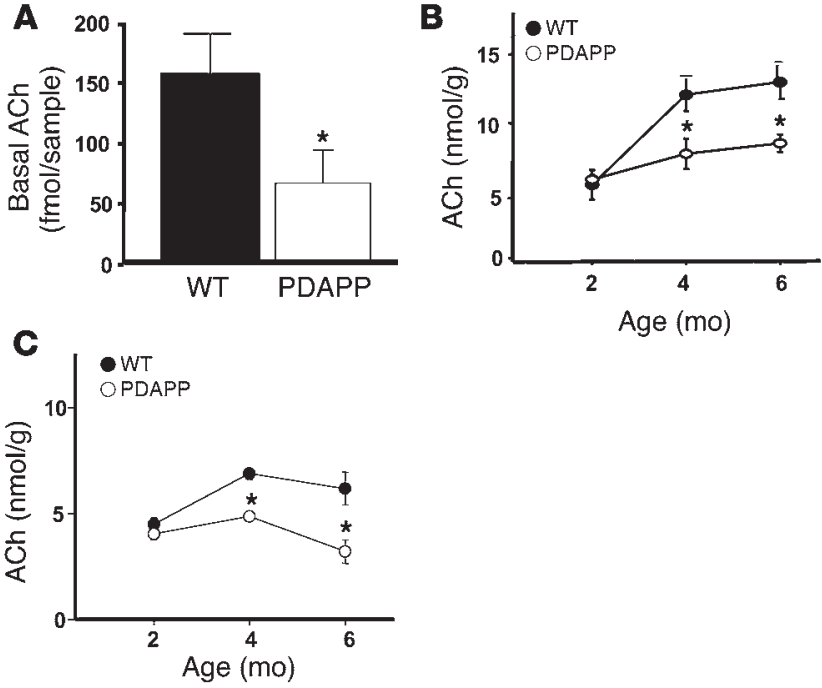

lowed by Western blot analysis to determine that $\mathrm{A} \beta$ could directly associate with ChT-1 and potentially interfere with its normal physiological role. Finally, acute treatment of PDAPP mice with the anti-A $\beta$ antibody $\mathrm{m} 266$ is able to fully restore cholinergic tone and impaired habituation learning, suggesting that if the same soluble "cholinotoxic" species of $A \beta$ are involved with memory deficits associated with early AD or mild cognitive impairment, then passive administration with anti-A $\beta$ antibodies may have similar beneficial effects in the clinic.

\section{Results}

Basal release and tissue levels of hippocampal ACh are reduced in PDAPP mice. We first directly assessed ACh release in the hippocampus of young (pre-plaque) PDAPP and WT mice under steady-state conditions using in vivo microdialysis and found that basal levels of hippocampal ACh release were markedly reduced in PDAPP mice compared with age- and background-matched WT controls (Figure $1 \mathrm{~A} ; P<0.05)$. Since in vivo microdialysis measures extracellular concentrations of ACh, we also determined the tissue levels of ACh in hippocampal and cortical homogenates from these mice. Similar concentrations of ACh were measured in young mice (2 months of age), but tissue levels of ACh were significantly reduced in PDAPP mice at older ages ( $>4$ months), confirming that the reduced level of ACh release from the hippocampus as measured with in vivo

\section{Figure 2}

Hippocampal ACh release in PDAPP mice is restored to WT levels after anti-A $\beta$ treatment. (A) Hippocampal ACh release in WT and PDAPP transgenic mice following exposure to a novel environment (Novelty) and after treatment of PDAPP mice with the anti-A $\beta$ antibody m266 (PDAPP + m266; $500 \mu$ i.p.). The arrows indicate the time at which mice were placed into a novel environment (novelty) and back into their home cage. (B) Release of ACh from the hippocampus of WT and PDAPP mice following administration of scopolamine $(0.3 \mathrm{mg} / \mathrm{kg}$ i.p.) and after administration of m266. $n=7-10$ mice per group, $4-6$ months of age. (C) Extracellular levels of choline in brain measured by in vivo microdialysis from WT and PDAPP mice. $n=5$ mice per group, 4-6 months of age. (D) Basal levels of hippocampal ACh release in WT, PDAPP, and PDAPP mice administered m266. $n=7-10$ mice per group, 4-6 months of age. ${ }^{\star} P<0.05$ versus WT; ${ }^{*} P<0.05$, PDAPP versus PDAPP + m266.

\section{Figure 1}

Hippocampal ACh release and tissue levels are reduced in PDAPP transgenic mice. (A) Basal levels of hippocampal ACh release measured by in vivo microdialysis from WT and PDAPP transgenic mice ( $n=7-10$ mice per group, 4-6 months of age). (B and C) Tissue levels of ACh from the hippocampus (B) and cortex (C) of PDAPP and WT mice ( $n=6$ mice per group). ${ }^{*} P<0.05$ versus WT.

microdialysis mirrored those that were measured directly in tissue homogenates (Figure 1, B and C; $P<0.05$ ).

Evoked release of $A C h$ is dysregulated in PDAPP mice. We next determined the functional responsiveness of hippocampal cholinergic neurons in PDAPP and WT mice following a pharmacological (systemic administration of scopolamine) or physiological (exposure to a novel environment) challenge. Both conditions have been shown to increase hippocampal ACh efflux and to constitute a reliable index of functional responsiveness of the cholinergic system (18). As previously reported, when WT mice are placed into a novel environment, a situation expected to evoke a physiological release of ACh, hippocampal ACh efflux increases modestly ( $50 \%)$ and then returns to baseline levels within 60 minutes (18). By contrast, when PDAPP mice were exposed to the same novel environment, the level of hippocampal ACh release was markedly elevated and prolonged, increasing by more than 2.5 -fold for nearly 90 minutes,
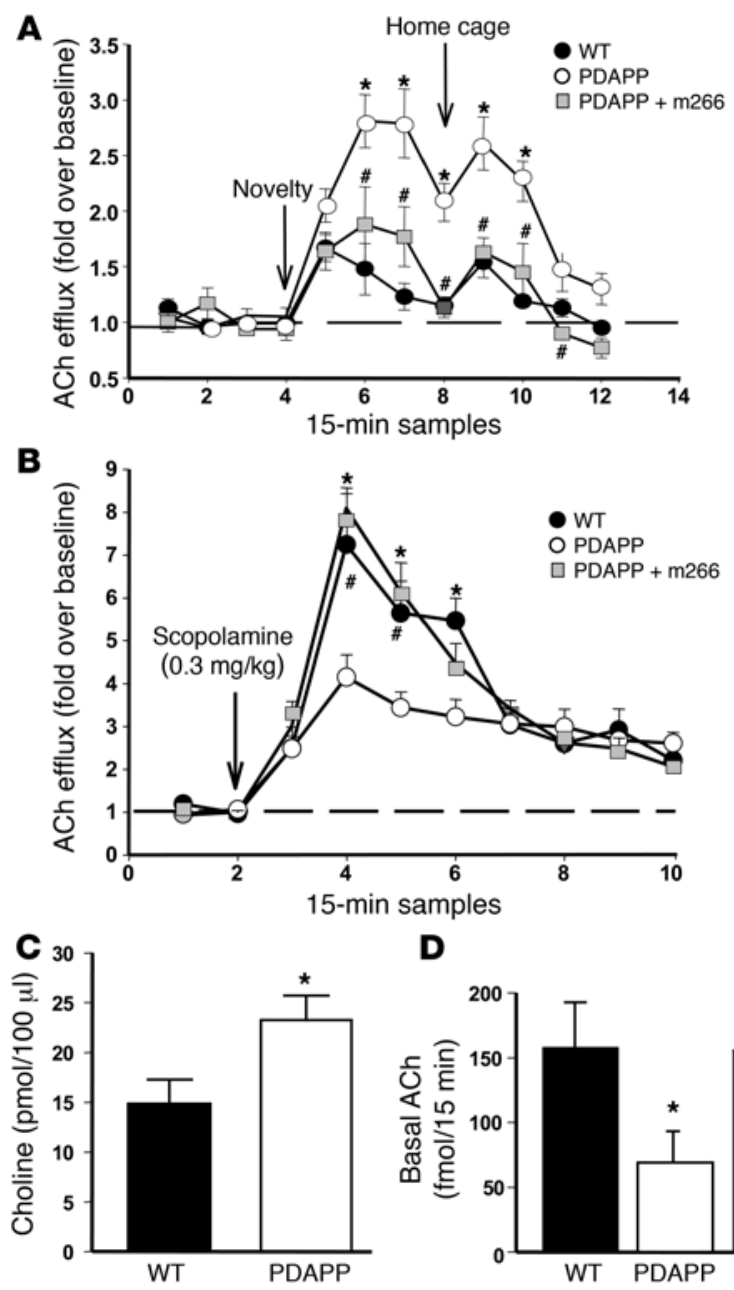

D

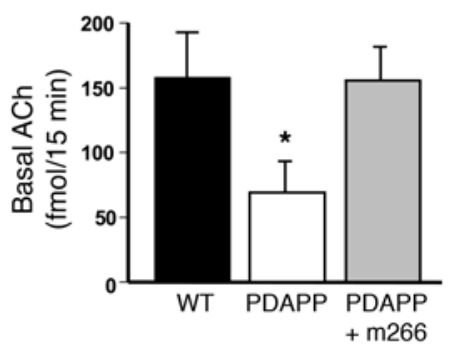




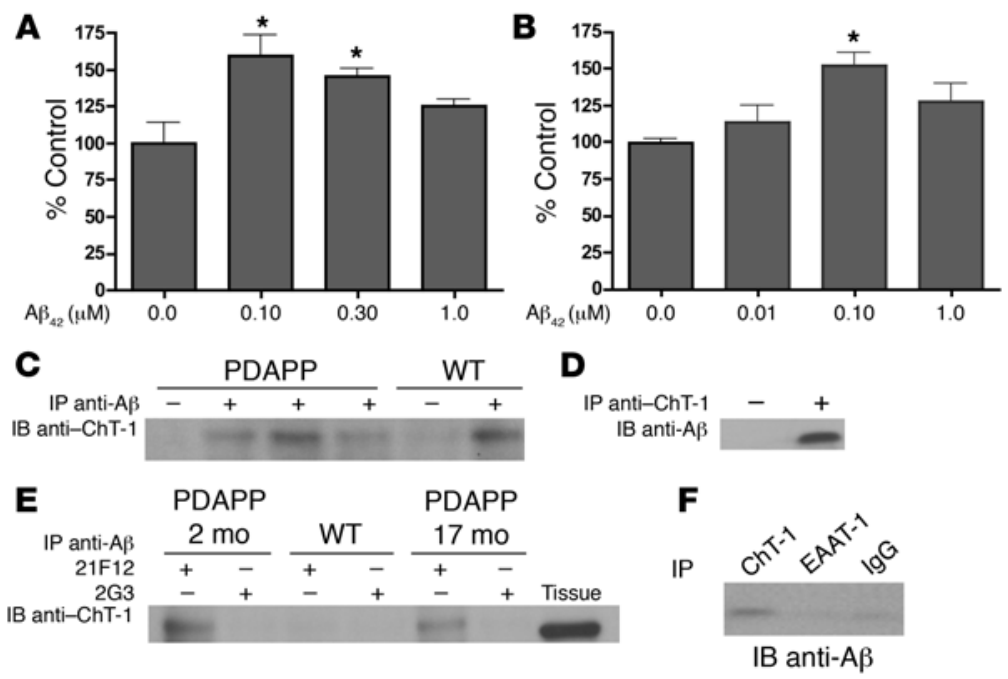

Figure 3

A $\beta$ interacts with ChT-1. (A and B) High-affinity choline uptake into rat synaptosomes (A) and a cell line expressing the human ChT-1 (B) was significantly increased after exposure to $A \beta_{42}$. Data are the percent of choline uptake in untreated, control samples (mean \pm SEM of triplicate values) from 1 representative experiment, which was repeated 2-3 times with similar results. ${ }^{*} P<0.05$. (C) ChT-1 was coimmunoprecipitated from hippocampal extracts by the anti-A $\beta$ antibody $4 \mathrm{G} 8$ followed by Western blot analysis with an anti-ChT-1 antibody. (D) The A $\beta$ peptide was coimmunoprecipitated from hippocampal extracts prepared from PDAPP transgenic mice by an anti-ChT-1 antibody followed by Western blot analysis with biotinylated anti-A $\beta$ antibodies $21 \mathrm{~F} 12$ and $2 \mathrm{G} 3$. (E) The anti-A $\beta_{42}$ antibody $21 \mathrm{~F} 12$, but not the anti-A $\beta_{40}$ antibody $2 \mathrm{G} 3$, coimmunoprecipitated $\mathrm{ChT}-1$ from hippocampal extracts prepared from PDAPP transgenic mice followed by Western blot analysis with an anti-ChT-1 antibody. (F) Neither an irrelevant IgG nor an anti-glutamate 1 transporter antibody (EAAT-1) coimmunoprecipitated $A \beta$ from hippocampal extracts prepared from PDAPP mice, whereas $A \beta$ was readily detected following immunoprecipitation with an antibody directed toward ChT-1 followed by Western blot analysis with an anti-A $\beta$ antibody.

compared with the levels of hippocampal ACh release observed in WT mice measured under identical conditions (Figure 2A; $P<0.05)$. Following administration of scopolamine $(0.3 \mathrm{mg} / \mathrm{kg}$ i.p.), a pan-muscarinic receptor antagonist, the level of ACh release (resulting from blockade of inhibitory muscarinic receptors) from the hippocampus of PDAPP mice was significantly reduced compared with similarly treated WT mice (Figure $2 \mathrm{~B} ; P<0.05$ ). Under all parameters measured (i.e., basal conditions), and following physiological (exposure to novelty) or pharmacological (following administration of scopolamine) stimuli, the efflux of ACh from the hippocampus of PDAPP mice was significantly different from that of age- and background-matched WT mice (Figure 2).

Extracellular levels of choline are increased in the hippocampus of PDAPP mice. Could the reduced extracellular and tissue levels of ACh be due to reduced availability of the ACh precursor choline? To address this possibility, we determined the level of extracellular choline using in vivo microdialysis. To our surprise, extracellular choline was significantly increased in PDAPP mice compared with age-matched WT controls (Figure 2C; $P<0.05$ ), thus confirming that reduced extracellular levels of the ACh precursor could not account for the reduced level of ACh measured in either tissue homogenates or the extracellular fluid with in vivo microdialysis (see Discussion).

$A \beta$ directly interacts with ChT-1. One possible explanation for the significant increase in extracellular levels of choline is that the
A $\beta$ peptide interacts directly with ChT-1, effectively blocking efficient transport and ACh biosynthesis. To investigate this possibility, we prepared synaptosomes from rat hippocampi and determined high-affinity choline transport following exposure to human $\mathrm{A} \beta_{42}$ (Figure 3A). There was a significant increase in choline uptake when rat synaptosomes where exposed to $\mathrm{A} \beta_{42}(100 \mathrm{nM} ; P<0.05)$. Since nerve terminal preparations contain elements of synaptic vesicles and $A \beta$ has been observed to have effects on multiple intracellular systems including synaptic vesicles, we next determined whether high-affinity choline uptake would be affected when cells expressing human ChT-1 were exposed to human $A \beta_{42}$ (Figure $3 B$ ). Similar to the results obtained when rat synaptosomes were exposed to $A \beta_{42}$, there was a significant increase in choline uptake even when cells expressing human ChT- 1 were exposed to very low concentrations of $A \beta_{42}$ (100 nM; $P<0.05)$ for a relatively short (15-minute) period of time. The reverse peptide $\left(A \beta_{42-1}\right)$ was without effect while the $A \beta$-dependent increase in highaffinity choline uptake occurred following exposure to either freshly prepared or aged $A \beta_{42}$ peptide (data not shown). We next used coimmunoprecipitation to determine which if any species of $A \beta$ is associated with ChT-1. When hippocampal homogenates prepared from PDAPP mice were coimmunoprecipitated with an anti-A $\beta$ antibody, 4G8, an approximately $70-\mathrm{kDa}$ band representing ChT-1 was apparent (Figure 3C). Conversely, an approximately $4-\mathrm{kDa}$ band representing $A \beta$ was clearly evident when hippocampal homogenates from PDAPP mice were coimmunoprecipitated with an anti-ChT-1 antibody followed by immunoblotting with the C-terminal-specific anti$A \beta$ antibodies $21 F 12$ and $2 \mathrm{G} 3$ (Figure $3 \mathrm{D}$ ). No $A \beta$ was detected when hippocampal homogenates from WT mice were coimmunoprecipitated with an anti-ChT-1 antibody and probed with anti-A $\beta$ antibodies (data not shown). ChT- 1 was, however, coimmunoprecipitated from hippocampal homogenates prepared from WT mice by the anti-A $\beta$ antibody 4G8 (Figure 3C). Since our initial coimmunoprecipitation experiments utilized an anti-A $\beta$ antibody that recognizes the mid-domain region of the $A \beta$ peptide and would thus coimmunoprecipitate both $A \beta_{40}$ and $\mathrm{A} \beta_{42}$, we next sought to determine whether either or both species of $A \beta$ could interact with ChT- 1 . Again we employed coimmunoprecipitation of $A \beta$ utilizing anti-A $\beta$ antibodies selective for $A \beta$ peptides ending in either 40 (2G3) or 42 (21F12) amino acids. Similar to the results obtained following coimmunoprecipitation with the anti-A $\beta$ antibody 4G8, ChT- 1 was detected when hippocampal homogenates from PDAPP mice were coimmunoprecipitated with $A \beta_{42}$-selective antibody $21 \mathrm{~F} 12$, but not with $\mathrm{A} \beta_{40}$-selective antibody $2 \mathrm{G} 3$, followed by immunoblotting with an anti-ChT-1 antibody (Figure 3E). ChT-1 was not detected when hippocampal homogenates from WT mice were coimmunoprecipitated with either C-terminal-selective antibody (Figure 3E).

Since the highly hydrophobic nature of $A \beta$ could result in a nonspecific association with ChT-1, we performed coimmunoprecipitation experiments with an antibody directed against the glutamate-1 transporter as well as with an irrelevant IgG. We did not detect any $\mathrm{A} \beta$ when hippocampal homogenates from PDAPP mice 


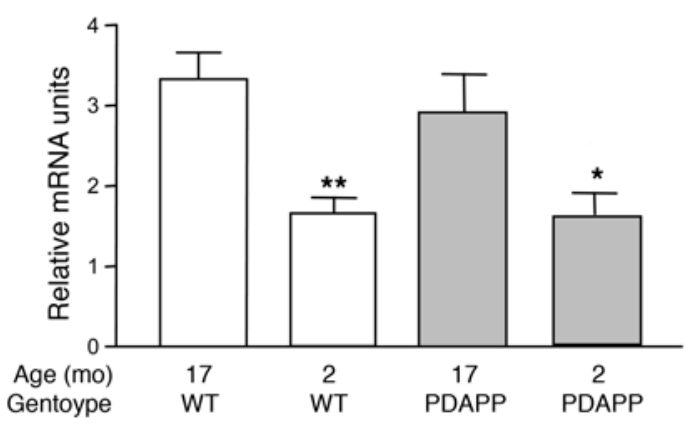

Figure 4

Relative mRNA level of ChT-1 in the hippocampus of young (2 months of age) and old (17 months of age) WT and PDAPP transgenic mice as determined by real-time quantitative PCR amplification. $n=5$ mice per group. ${ }^{* \star} P<0.01,{ }^{*} P<0.05$ versus respective 17 -month-old groups.

were immunoprecipitated with an anti-glutamate- 1 transporter antibody or irrelevant IgG followed by probing for the presence of $\mathrm{A} \beta$ (Figure 3F).

High-affinity choline uptake into hippocampal synaptosomes is increased in PDAPP mice. Since the rate-limiting step in ACh biosynthesis is the uptake of extracellular choline by the neuronal high-affinity ChT-1, we next determined the level of ChT-1 mRNA in PDAPP and WT mice, as a decrease in ChT-1 expression could explain the reduced basal levels of ACh and higher levels of extracellular choline in brain. There was no significant difference in the level of hippocampal ChT-1 mRNA between PDAPP and WT controls as determined by real-time quantitative PCR (Figure 4) at either 2 or 17 months of age. Interestingly, however, we found an age-dependent increase in the level of ChT-1 mRNA in both WT and PDAPP mice (Figure 4). To further explore the functional consequences of $\mathrm{A} \beta$ overexpression on high-affinity choline transport, we measured high-affinity choline uptake by ChT-1 in hippocampal synaptosomes from WT and PDAPP mice. The hippocampus was chosen because of the well-characterized cholinergic innervation to this brain region as well as the presence of high levels of the A $\beta$ peptide in PDAPP mice $(14,19-22)$. A kinetic analysis of highaffinity choline uptake in PDAPP or WT hippocampal synaptosomes revealed a significant increase $(50.9 \% ; P<0.05)$ in the $V_{\max }$ of choline uptake in PDAPP versus WT control mice, with no measurable change in apparent $K_{\mathrm{m}}$ (Table 1 ).

Anti-A $\beta$ antibody treatment restores the hippocampal ACh release profile in PDAPP mice. We next investigated whether acute treat-

\section{Figure 5}

Hippocampal ACh release is unaltered after treatment with other IgGs. (A) Hippocampal release of ACh in WT and PDAPP transgenic mice following exposure to a novel environment and 24 hours after the administration of an irrelevant IgG (PDAPP + IgG; $500 \mu$ g i.p.) or the anti-A $\beta$ antibody 3D6 (PDAPP + 3D6; $500 \mu$ g i.p.). (B) Release of ACh from the hippocampus of PDAPP and WT mice following administration of scopolamine $(0.3 \mathrm{mg} / \mathrm{kg}$ i.p.) 24 hours after the administration of an irrelevant IgG or the anti-A $\beta$ antibody 3D6. No significant difference between treated PDAPP and untreated PDAPP mice were observed in either A or B. (C) Basal levels of hippocampal ACh release from WT and PDAPP mice 24 hours following administration of an irrelevant IgG or the anti-A $\beta$ antibody $3 \mathrm{D} 6$. ${ }^{*} P<0.05$ versus $\mathrm{WT}$ and PDAPP + 3D6. $n=3-10$ mice per group, 4-6 months of age. ment with the high-affinity anti-A $\beta$ antibody $\mathrm{m} 266$ could restore hippocampal ACh homeostasis, since we have previously demonstrated that acute treatment of PDAPP mice with $\mathrm{m} 266$ was able to reverse memory deficits without any effects on brain $A \beta$ burden and that $\mathrm{m} 266$ binds with high affinity to soluble forms of $A \beta(13,23)$. PDAPP mice were treated with a single dose of $\mathrm{m} 266$ $(500 \mu \mathrm{g}) 24$ hours prior to the establishment of baseline ACh levels. ACh release from the hippocampi following scopolamine administration in PDAPP mice administered m266 under basal conditions were not significantly different from untreated WT mice but were significantly different from untreated PDAPP mice (Figure 2, B and D; $P<0.05$ ). Similarly, when PDAPP mice were exposed to a novel environment following $\mathrm{m} 266$ administration, the magnitude and duration of hippocampal ACh release were not significantly different from that of WT mice but were again significantly different from untreated PDAPP mice (Figure 2A; $P<0.05)$. To determine the specificity of $\mathrm{m} 266$ treatment, we utilized an irrelevant IgG (isotype matched to m266) antibody as well as an additional anti-A $\beta$ antibody (3D6) that recognizes the $\mathrm{N}$ terminus of the human $\mathrm{A} \beta$ peptide (20). Neither the irrelevant IgG nor 3D6 were able to reverse or normalize hippocampal ACh release in response to a novel environment or following scopolamine administration (Figure 5, A and B). However, treatment with $3 \mathrm{D} 6$, but not with the irrelevant $\mathrm{IgG}$, was able to restore
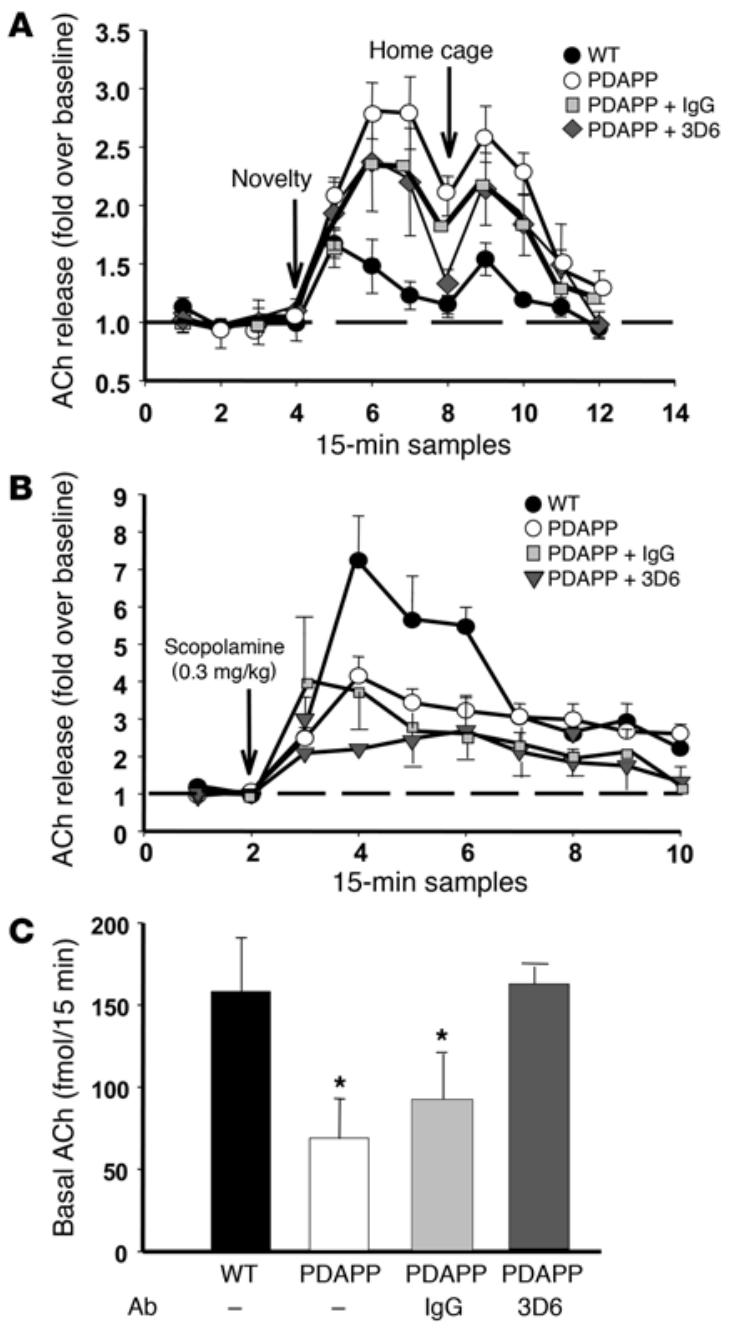
Table 1

Kinetics of choline uptake into hippocampal synaptosomes

\begin{tabular}{lcc}
\hline & $\boldsymbol{V}_{\max }(\mathbf{p m o l} / \mathbf{m g} / \mathbf{5} \mathbf{~ m i n})$ & $\boldsymbol{K}_{\mathrm{m}}(\boldsymbol{\mu M})$ \\
WT & $26.61 \pm 5.45$ & $0.85 \pm 0.14$ \\
PDAPP & $40.16 \pm 7.87^{\mathrm{A}}$ & $1.23 \pm 0.33^{\mathrm{B}}$ \\
$\mathrm{PDAPP}+\mathrm{m} 266$ & $19.97 \pm 6.62^{\mathrm{C}}$ & $0.50 \pm 0.28^{\mathrm{B}}$ \\
\hline
\end{tabular}

Values are mean \pm SEM of $2-5$ experiments. Alncrease of $50.9 \%$ compared with WT controls $(P<0.05)$. ${ }^{B}$ No significant difference compared with WT mice. 'Decrease of $50.2 \%$ compared with untreated PDAPP transgenic mice.

basal hippocampal ACh release following administration to PDAPP mice (Figure 5C). We also determined high-affinity choline uptake in hippocampal synaptosomes prepared from PDAPP mice that had been treated with $\mathrm{m} 266$ prior to preparation of the crude synaptosome fraction (22). When PDAPP mice were administered $\mathrm{m} 26624$ hours prior to measuring choline uptake, the $V_{\max }$ values were not significantly different between PDAPP and WT control mice (Table 1).

Habituation learning in PDAPP mice is restored following $m 266$ treatment. Since habituation of locomotor activity to a novel environment provides a measure of learning processes related to primal memory formation and since ACh levels are known to increase when rodents are placed into a novel environment, we measured habituation to a novel environment after PDAPP mice were treated with m266 $(18,24)$. As predicted, young (pre-plaque) PDAPP mice, which display cholinergic hyperresponsiveness to novelty, failed to habituate to a novel environment (data not shown). Following m266 treatment, however, PDAPP mice habituated to their novel environment in a manner similar to their WT counterparts (Figure 6). By contrast, when PDAPP mice were treated with 3D6, they, like untreated PDAPP mice, failed to habituate to a novel environment (Figure 6).

\section{Discussion}

Recently, $A \beta_{1-42}$ and the $\alpha 7$ nicotinic ACh receptor $(\alpha 7)$ was reported to form a high-affinity, stable complex in vitro as well as colocalize to neuritic plaques in $\mathrm{AD}$ brain, suggesting that $\mathrm{A} \beta_{1-42}$ may influence normal cholinergic neurotransmission by directly modulating the $\alpha 7$ (25). To our knowledge, however, ours is the first demonstration that the $\mathrm{A} \beta$ peptide(s) directly associate with ChT-1 and perturb its physiological function, thereby impairing cholinergic neurotransmission in vivo. Using a well-characterized transgenic mouse model of AD - the PDAPP mouse - combined with in vivo microdialysis, we quantified ACh release from the hippocampi of awake and freely moving animals and observed a significant reduction in ACh release in PDAPP mice under basal conditions and a pronounced dysregulation of cholinergic neurotransmission in response to either pharmacological or physiological stimuli, which assess and are associated with normal cognitive function. The reduced hippocampal release and basal tissue levels of ACh were not due to reduced mRNA levels of ChT-1 or extracellular levels of the ACh precursor choline. In contrast, there was a significant increase in the rate of high-affinity choline uptake when synaptosomes were prepared from PDAPP mice that most likely represents a compensatory change in response to a deficient cholinergic synapse. Furthermore we determined by coimmunoprecipitation experiments that $\mathrm{A} \beta$ could directly associate with ChT-1 and thereby interfere with its intracellular trafficking, localization, and normal physiological functions.

Administration of the anti-A $\beta$ antibody m 266 to PDAPP mice restored hippocampal ACh release to near WT levels when measured under either basal conditions or after mice were exposed to a novel environment. More dramatic, however, was the nearly complete restoration of hippocampal ACh release when PDAPP mice were administered $\mathrm{m} 266$ prior to scopolamine administration. Additionally, the rate of choline uptake into hippocampal synaptosomes prepared from PDAPP mice following administration of m266 was nearly completely restored to that observed in WT mice. Taken together, our results suggest that the anti-A $\beta$ antibody $\mathrm{m} 266$ can bind and neutralize cholinotoxic species of the $A \beta$ peptide since neither an irrelevant IgG nor another anti-A $\beta$ antibody, 3D6, affected hippocampal ACh release when PDAPP mice were placed in a novel environment or administered scopolamine.

Since habituation of locomotor activity in a novel environment provides a measure of primal learning and memory processes and is dependent on an integral cholinergic circuit, we used this readily quantifiable behavior to examine the effect of acute treatment with m266 (24). As previously reported, PDAPP mice fail to habituate to a novel environment to the same extent as WT mice (17). However, following acute treatment with the anti-A $\beta$ antibody $\mathrm{m} 266$, this behavioral deficit was reversed. Given previous reports demonstrating transient elevations in brain ACh levels when rodents are placed in a novel environment, our data suggest that "normalizing" hippocampal ACh release by acute treatment with $\mathrm{m} 266$ is most likely causally related to restored habituation to a novel environment (24). Interestingly, the profile of hippocampal ACh release we observed in PDAPP mice was similar to that of mice lacking M2 muscarinic receptors, suggesting that $A \beta$ may also influence muscarinic inhibitory autoreceptors leading to inefficient regulation of synaptically released ACh (18).

Since we used young PDAPP mice, which have little to no immunoreactive $A \beta$ deposits or frank amyloid (thioflavine-S-positive) deposition, our results strongly implicate soluble forms of the $A \beta$

\section{Figure 6}

Habituation learning in a novel open field in PDAPP mice is normalized after treatment with m266. (A) PDAPP transgenic mice exhibited increased locomotor activity, which was reduced following treatment with the anti-A $\beta$ antibody m266 (500 $\mu$ g i.p.) but not following treatment with the antiA $\beta$ antibody 3D6 (500 $\mu$ g i.p.). (B) Accumulated locomotor activity per 60 -minute session. ${ }^{* \star *} P<0.001 . n=20$ mice per group, 4-6 months of age.
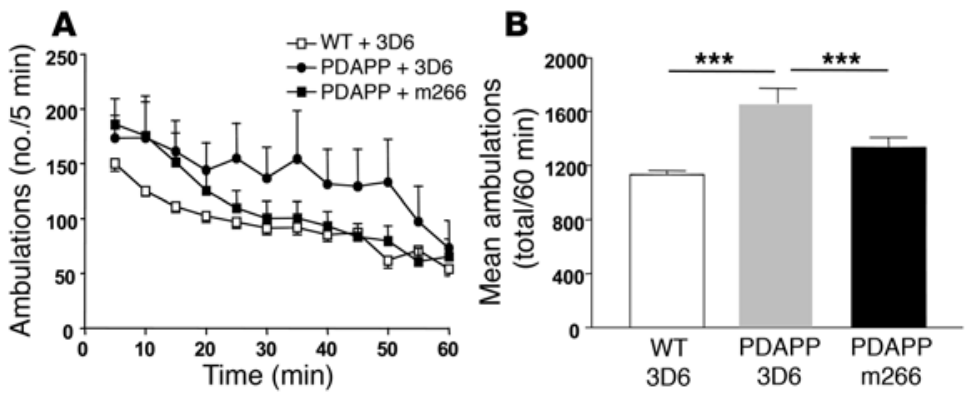
peptide(s) in disrupting basal and on-demand hippocampal ACh release, both of which are likely required for normal memory function (1). Indeed there is now ample in vitro evidence that suggests that soluble forms of $A \beta$ inhibit long-term potentiation as well as dampen neuronal activity following depolarization $(7,26,27)$. Recently, oligomeric soluble forms of human $A \beta$ were reported to disrupt complex learning behavior in rats following intracerebroventricular administration (28). Moreover, in vivo evidence from various APP transgenic mouse models suggests that $A \beta$-directed immunotherapy (either passive or active immunization) can effectively reverse memory deficits without any measurable effect on deposited (insoluble) A $\beta(13,29)$.

Our results do not eliminate the possibility that other fragments of APP may modulate hippocampal ACh efflux. Since certain forms of secreted APP have memory-enhancing effects, it may be possible that other fragments of APP derived from proteolytic cleavage would have differential effects on cholinergic neurotransmission; this possibility will also need further investigation (30). However, the fact that both the dysregulation in ACh release and impaired habituation learning were rapidly reversed by the anti-A $\beta$ antibody $\mathrm{m} 266$ strongly argues for a pivotal role of the $\mathrm{A} \beta$ peptide as a cholinotoxic substance. One intriguing possibility is the potential for soluble forms of the $A \beta$ peptide(s) to interact and/or directly modulate ChT- 1 since the activity of the ACh biosynthetic enzyme choline acetyltransferase was not decreased in brain samples from PDAPP mice (K.R. Bales and S.M. Paul, unpublished observations) and patients with mild AD (31). Moreover, recent reports suggest that $A \beta$ can regulate the cell surface expression of glutamate receptors, thereby affecting normal glutamatergic neurotransmission (32).

High-affinity uptake of choline at cholinergic nerve terminals is widely believed to be the rate-limiting step in the biosynthesis of ACh, and a significant increase in high-affinity choline transport was reported to occur in synaptosomes prepared from rapid autopsy $\mathrm{AD}$ brain tissue; this most likely reflects a compensatory response initiated by remaining cholinergic neurons due to severe cholinergic deafferentation (33). Additionally, deletion of 1 choline acetyltransferase allele in mice does not result in a corresponding decrease in ACh biosynthesis since enhanced recruitment and expression of ChT-1 results in normal ACh biosynthesis (34). Our finding of a significant increase $(-50 \%)$ in the rate of choline uptake into synaptosomes prepared from PDAPP mice appears inconsistent with our observation of reduced basal/tissue levels of ACh. We hypothesize that enhanced enrichment of ChT-1 to a synaptic vesicular pool in PDAPP mice is the result of a compensatory response driven by the inability of the cholinergic neuron to effectively transport choline to the intracellular space for subsequent ACh biosynthesis. Additionally, the significant increase in extracellular choline levels we observed in PDAPP mice suggests that A $\beta$ may in some way interfere with efficient ChT-1-mediated transport of choline and/or block normal cell surface redistribution of ChT-1, thereby resulting in impaired ACh biosynthesis and reduced tissue levels (35). Further studies will be required to fully delineate the exact interaction between ChT- 1 and $A \beta$; however, our results that $\mathrm{A} \beta$ directly associates with ChT-1, potentially impeding ACh biosynthesis, bolster the hypothesis of selective cholinergic membrane "autocannibalization" under sustained ACh demand, which may explain the increased vulnerability of cholinergic neurons in AD brain (36). Additionally our data do not eliminate the possibility that certain species of $\mathrm{A} \beta$ (especially $A \beta_{40}$ ) may subserve a physiologically relevant role, while other species (especially $A \beta_{42}$ ) may exert a more pathological role on cholinergic neurotransmission. Interestingly, recent reports have demonstrated that $\alpha$-synuclein, another brain protein prone to aggregate in Parkinson disease, interacts with the dopamine transporter, suggesting that abnormal protein aggregation in general may interrupt neurotransmission by interfering with normal transporter function (37). Finally, it is also feasible that up- and/or downregulation of pre- and post-synaptic muscarinic receptors could influence, in an $A \beta$-dependent fashion, the efflux of ACh from the hippocampus.

Although the cholinergic neurotransmitter system is neither the only nor the first neurotransmitter system affected in $\mathrm{AD}$, it is widely believed that cholinergic deafferentation in $\mathrm{AD}$ brain contributes to the memory decline associated with disease progression. Further work will be required to address the exact mechanism(s) by which $A \beta$ influences cholinergic neurotransmission and neurodegeneration; however, our data clearly demonstrate a direct cholinotoxic effect of the A $\beta$ peptide(s) on hippocampal ACh release in PDAPP mice that was rapidly reversed by treatment with the anti-A $\beta$ antibody $\mathrm{m} 266$. Taken together, our findings suggest that $\mathrm{A} \beta$-mediated disruption of cholinergic neurotransmission and memory can occur in the absence of overt neurodegeneration and that administration of certain anti-A $\beta$ antibodies may reverse early memory impairment especially in patients with mild cognitive impairment or early AD.

\section{Methods}

Transgenic mice. The PDAPP transgenic mice used in this study were homozygous mice derived from a heterogeneous background comprising the strains C57BL/6J, DBA/2J, and Swiss-Webster (14). Young (4-6 months of age) female PDAPP mice as well as age- and backgroundmatched WT controls were used for all in vivo microdialysis studies. For determining open field activity in a novel environment, young PDAPP males (4-6 months of age) as well as background-, age- and gendermatched WT controls were assessed. All experiments utilizing animals were reviewed and approved by the Internal Animal Care and Use Committee of Lilly Research Laboratories.

In vivo microdialysis. Mice were deeply anesthetized with a mixture of chloral hydrate and pentobarbital, and a CMA/7 (2 $\mathrm{mm})$ dialysis probe (CMA Microdialysis) was implanted unilaterally into the hippocampus (AP, -3.3; ML , 3.1; DV, -4.2). Mice were allowed to recover in their home cages for more than 24 hours and on the morning of the experiments were transferred to a Plexiglas cylinder $(15 \mathrm{~cm} \times 25 \mathrm{~cm})$ with clean bedding. The dialysis probe was connected to a liquid swivel (TCS-2; SciPro Inc.) microdialysis instrument. Perfusate was delivered at a rate of $1.5 \mu \mathrm{l} / \mathrm{min}$, and samples were analyzed in-line with HPLC coupled to an electrochemical detection (EC) system as previously described (18). The perfusate was a modified artificial Ringer's solution supplemented with $0.33 \mu \mathrm{M}$ neostigmine to prevent enzymatic degradation. The mice were allowed to acclimate to this setting for 4 hours, during which time dialysate samples were collected every 15 minutes. Little variation (<10\%) occurred in the ACh signal during this period, and baseline levels of ACh efflux were established as the mean of 5 15-minute samples prior to any subsequent manipulation. When mice were exposed to a novel environment, they were transferred into a new cage that was approximately the same size but markedly different in color (dark red versus clear) and texture (thick rubber without bedding versus Plexiglas with a bedding-covered floor). The mice remained in this novel environment for 1 hour, during which time dialysate samples 
were collected and analyzed as described above. After 1 hour, the mice were transferred back into their original testing cage (Plexiglas cylinder), and samples were collected for an additional 1 hour, during which time ACh levels returned to baseline. For pharmacological manipulation, mice were injected with scopolamine $(0.3 \mathrm{mg} / \mathrm{kg}$ i.p.), and samples were collected for an additional 3 hours. To assess the effects of various IgGs (anti-A $\beta$ antibodies $\mathrm{m} 266$ and 3D6 and irrelevant IgG, $500 \mu$ i.p.), the same experimental paradigm described above for collecting and analyzing ACh efflux was followed, except that the mice were administered IgG 24 hours prior to the initial baseline collection and 24 hours after the probes were implanted. Extracellular choline concentrations were assessed by in vivo microdialysis essentially as described above. A modified Ringer's solution devoid of neostigmine was perfused, and 5 -minute samples were collected and analyzed online with HPLC-EC (38).

Tissue ACb levels. ACh levels in tissue homogenates were determined essentially as described previously (39). Briefly, mice were quickly decapitated, and brain regions were dissected and frozen on dry ice within 30 seconds. The tissues were homogenized in 10 volumes of $0.1 \mathrm{M}$ trichloroacetic acid containing $10 \mu \mathrm{M}$ acetylthiocholine as an internal standard. After centrifugation at $10,000 \mathrm{~g}, 20-\mu \mathrm{l}$ aliquots of the supernatant were analyzed for ACh with HPLC-EC (39).

Choline uptake. Choline uptake into hippocampal synaptosomes was measured as previously described (22). Briefly, hippocampi from 4- to 6-monthold PDAPP mice and WT controls were dissected and pooled ( $n=10-12$ per experiment). Crude synaptosomes were prepared by homogenizing the tissue in ice-cold buffer ( $0.32 \mathrm{M}$ sucrose, 10 strokes Dounce homogenizer AA068), and total protein content was determined (Pierce Biotechnology) from $\mathrm{P} 2$ pellets after centrifugation $(12,000 \mathrm{~g}$ for 20 minutes). Choline uptake was determined in triplicate by incubating $0.1-0.2 \mathrm{mg}$ of the crude synaptosomal protein in choline uptake buffer $(130 \mathrm{mM} \mathrm{NaCl}, 1.3 \mathrm{mM}$ $\mathrm{KCl}, 1.2 \mathrm{mM} \mathrm{MgSO}_{4}, 1.2 \mathrm{mM} \mathrm{KH} \mathrm{PO}_{4}, 10 \mathrm{mM}$ glucose, $10 \mathrm{mM}$ HEPES, and $2.2 \mathrm{mM} \mathrm{CaCl}, \mathrm{pH} 7.4)$ and $\left[{ }^{3} \mathrm{H}\right]$ choline $(86 \mathrm{Ci} / \mathrm{mmol} ; 10 \mathrm{nM}$; PerkinElmer), with final choline concentrations ranging $0.03-5 \mu \mathrm{M}$, for 5 minutes at $37^{\circ} \mathrm{C}$. Uptake was terminated by returning samples to ice for 5 minutes followed by aspiration onto polyethyleneimine-coated glass filters (PerkinElmer). The specificity of uptake was determined by subtracting choline uptake in the presence of hemicholinium $(10 \mu \mathrm{M}) . K_{\mathrm{m}}$ and $V_{\max }$ values were determined by nonlinear regression analysis with GraphPad Prism software (version 4.03; GraphPad Software Inc.). For determining the effects of $\mathrm{m} 266$ on high-affinity choline uptake, PDAPP mice were administered 500 $\mu \mathrm{g}$ m266 i.p. 24 hours prior to the preparation of synaptosomes. For the determination of high-affinity choline uptake into rat hippocampal nerve termini, the above protocol was followed except that hippocampi from 2-3 rats were used. A stable cell line expressing the human ChT-1 was generated following selection in hygromycin after transfection into 293 cells. For high-affinity choline uptake determinations, cells were plated onto polyL-lysine-coated Cytostar T plates (60,000 cells/100 $\mu \mathrm{l}$; Amersham Biosciences) and incubated at $37^{\circ} \mathrm{C}$ for 24 hours prior to determining choline uptake. For determination of high-affinity uptake, media was removed and replaced with choline uptake buffer with ${ }^{14} \mathrm{C}$-choline $(0.2 \mu \mathrm{Ci} / \mathrm{ml} ; 3.75 \mu \mathrm{M}$ final concentration) and incubated for 70 minutes at room temperature. The plates were counted in a MicroBeta counter (PerkinElmer) for 1 minute. Specific uptake was determined by subtracting uptake in the presence of hemicholinium $(10 \mu \mathrm{M})$. To determine choline uptake following exposure to human $A \beta_{1-42}$ (AnaSpec), ChT-1 cells or rat hippocampal synaptosomes were exposed to $A \beta(0.01-1 \mu \mathrm{M})$, which was diluted from a stock solution $(1 \mathrm{mM})$, and incubated for 15 minutes at $37^{\circ} \mathrm{C}$. Choline uptake was then determined as described above

Coimmunoprecipitation. Hippocampi from PDAPP transgenic mice were homogenized in buffer ( $1 \%$ Nonidet P-40, $0.1 \%$ SDS, $50 \mathrm{mM}$ Tris $\mathrm{pH}$ 8.0,
$50 \mathrm{mM} \mathrm{NaCl}, 0.05 \%$ deoxycholate, and protease inhibitor), and protein concentrations were determined (Pierce Biotechnology). Hippocampal extracts $(250 \mu \mathrm{g})$ were diluted in $250 \mu \mathrm{l} \mathrm{PBS} / 0.2 \%$ BSA with antibodies $(2.5 \mu \mathrm{g}$; anti-A $\beta, 2 \mathrm{G} 3$, and $21 \mathrm{~F} 12$, all from Senetek; 4G8 from Signet Lab Inc.; anti-ChT-1, anti-glutamate-1 transporter from Chemicon International; irrelevant IgG from Harlan) for 8 hours at $4{ }^{\circ} \mathrm{C}$ on a rocking platform. Samples were incubated for an additional 16 hours after the addition of BSA (1\%) blocked Protein G beads (100 $\mu$ l; Pierce Biotechnology). The beads were washed 3 times in PBS $(500 \mu \mathrm{l})$, and proteins were eluted with sample loading buffer ( $30 \mu \mathrm{l}$; Novex, Invitrogen Corp.) prior to size fractionation (4-12\% Bis Tris gel; Bio-Rad). Proteins were then transferred onto PVDF membranes (Bio-Rad) and probed with an anti-ChT-1 antibody (Chemicon International) or anti-A $\beta$ antibodies $(21 \mathrm{~F} 12,2 \mathrm{G} 3)$ which were biotinylated. The signal was visualized with enhanced chemiluminescence (Pierce Biotechnology).

Habituation learning. Locomotor activity as a measure of habituation to a novel environment was assessed in photocell activity cages (San Diego Instruments), which consisted of a standard plastic rodent cage $(24 \mathrm{~cm} \times$ $45.5 \mathrm{~cm} \times 15.5 \mathrm{~cm}$ ) surrounded by a stainless steel frame. Locomotor activity frames consisted of 7 infrared photocell beams located across the long axis of the frame, raised $2 \mathrm{~cm}$ above the floor and placed $5.5 \mathrm{~cm}$ apart. The number of cage crossings, defined as consecutive interruptions of one beam followed immediately by interruption of an adjacent beam, were recorded by a computer system during consecutive 5-minute intervals for 60 minutes and used as a measure of spontaneous locomotion. Locomotor activity was evaluated in animals that were naive to the test apparatus but were habituated to the test room for approximately 1 hour prior to activity measurements. PDAPP or WT mice were injected with various IgGs $(500 \mu \mathrm{g}$ i.p.) or vehicle and assessed for locomotor activity 24 hours later.

$m R N A$ analysis. Total RNA was isolated from the hippocampus of 2- and 17 -month-old PDAPP mice $(n=6)$ following saline perfusion. Briefly, tissue was rapidly removed and stored on dry ice until homogenization. Total RNA was isolated using the RNeasy Mini Kit (QIAGEN) according to the manufacturer's guidelines. cDNA was generated using the Superscript (Invitrogen Corp.) preamplification system for first-strand synthesis according to the manufacturer's protocol and subsequently used (at 1:100 dilution) as the template for real-time quantitative PCR amplification. Primers specific for ChT-1 (forward, 5'-CCAGCTTTTGGGTGCCTG; reverse, 5'-TGTGGAAGCTCCAATAGCTCC; probe, 6FAM-TGATGGCTCTACCCGCCATATGC-TAMRA; 20-pmol and 5-pmol probe) were used. Amplification conditions were as follows: 10 minutes at $95^{\circ} \mathrm{C}$ followed by 40 cycles at $95^{\circ} \mathrm{C}$ for 15 seconds and $60^{\circ} \mathrm{C}$ for 1 minute in a PerkinElmer Applied Biosystems SDS7700 sequence detection system. Each sample was run in triplicate with non-reverse transcribed template serving as control. The relative level of ChT-1 mRNA was computed by the standard curve method utilizing GAPDH amplification for normalization.

Statistics. The basal values of ACh and choline in tissue and microdialysates in PDAPP and WT mice in response to antibody treatments were evaluated by 2-tailed Student's $t$ test (Figure 1A and Figure 2C), 2-way (genotype $\times$ age) ANOVA with repeated measures (Figure 1, B and C), or 1-way (treatment) ANOVA (Figure 2D and Figure 5C). The microdialysis data were also expressed and presented as the percent change from baseline (based on pretreatment values) and analyzed with a 2-way (treatment $x$ time) ANOVA (Figure 2, A and B, and Figure 5, A and B). In all cases, significant interactions were evaluated using the Newman-Keuls test for multiple comparisons. For high-affinity choline uptake experiments, the data were analyzed with a 1-way (treatment) or 2-way (treatment $x$ genotype) ANOVA and evaluated by the Newman-Keuls test for multiple comparison. For habituation learning, the mean ambulations during a 60-minute session were used for a 2-way (genotype $\times$ treatment) com- 
parison followed by the Tukey-Kramer test for multiple comparisons. $P<0.05$ was considered statistically significant.

\section{Acknowledgments}

The authors thank K. Svensson, P. May, C. Felder, and Y. Du for comments on the manuscript as well as D. Koger and C. Salhoff for technical assistance.

Received for publication October 12, 2005, and accepted in revised form January 3, 2006.

1. Perry, E., Walker, M., Grace, J., and Perry, R. 1999. Acetylcholine in mind: a neurotransmitter correlate of consciousness? Trends Neurosci. 22:273-280.

2. Bartus, R., Dean, R.L., Beer, B., and Lippa, A.S.1982. The cholinergic hypothesis of geriatric memory dysfunction. Science. 217:408-411.

3. Coyle, J.T., Price, D.L., and DeLong, M.R. 1983. Alzheimer's disease: a disorder of cortical cholinergic innervation. Science. 219:1184-1190.

4. Whitehouse, P.J., et al. 1981. Alzheimer disease: evidence for selective loss of cholinergic neurons in the nucleus basalis. Ann. Neurol. 10:122-126.

5. Hardy, J., and Selkoe, D.J. 2002. The amyloid hypothesis of Alzheimer's disease: progress and problems on the road to therapeutics. Science. 297:353-356.

6. Selkoe, D.J. 2001. Alzheimer's disease: genes, proteins, and therapy. Physiol. Rev. 81:741-766.

7. Walsh, D.M., et al. 2002. Naturally secreted oligomers of amyloid $\beta$ protein potently inhibit hippocampal long-term potentiation in vivo. Nature. 416:535-539.

8. Westerman, M.A., et al. 2002. The relationship between $A \beta$ and memory in the $\operatorname{Tg} 2576$ mouse model of Alzheimer's disease. J. Neurosci. 22:1858-1867.

9. McLean, C.A., et al. 1999. Soluble pool of A $\beta$ amyloid as a determinant of severity of neurodegeneration in Alzheimer's disease. Ann. Neurol. 46:860-866.

10. Lue, L.F., et al. 1999. Soluble amyloid $\beta$ peptide concentration as a predictor of synaptic change in Alzheimer's disease. Am. J. Pathol. 155:853-862.

11. Wang, J., Dickson, D.W., Trojanowski, J.Q., and Lee, V.M. 1999. The levels of soluble versus insoluble brain $\mathrm{A} \beta$ distinguish Alzheimer's disease from normal and pathologic aging. Exp. Neurol. 158:328-337.

12. Parvathy, S., et al. 2001. Correlation between $\mathrm{A} \beta \mathrm{x}-40-, \mathrm{A} \beta \mathrm{x}-42-$, and $\mathrm{A} \beta \mathrm{x}-43$-containing amyloid plaques and cognitive decline. Arch. Neurol. 58:2025-2032

13. Dodart, J.C., et al. 2002. Immunization reverses memory deficits without reducing brain $\mathrm{A} \beta$ burden in Alzheimer's disease model. Nat. Neurosci. 5:452-457.

14. Games, D., et al. 1995. Alzheimer-type pathology in transgenic mice overexpressing V717F beta-amy-

Address correspondence to: K.R. Bales or S.M. Paul, DC0533 Eli Lilly \& Co., Neuroscience Discovery Research, 355 E. Merrill Street, Indianapolis, Indiana 46285, USA. Phone: (317) 277-3061; Fax: (317) 277-6146; E-mail: KRBales@Lilly.com (K.R. Bales). Phone: (317) 277-8799; Fax: (317) 277-1125; E-mail: Paul_Steven_M@ Lilly.com (S.M. Paul).

K.R. Bales and E.T. Tzavara contributed equally to this work.

E.T. Tzavara's present address is: INSERM U-513, Créteil, France.

loid precursor protein. Nature. 373:523-527.

15. Irizarry, M., et al. 1997. Abeta deposition is associated with neuropil changes, but not with overt neuronal loss in the human amyloid precursor protein V717F (PDAPP) transgenic mouse. J. Neurosci. 15:7053-7059

16. German, D.G., et al. 2003. Cholinergic neuropathology in a mouse model of Alzheimer's disease. J. Comp. Neurol. 462:371-381.

17. Dodart, J.C., Mathis, C., Bales, K.R., and Paul, S.M 1999. Behavioral disturbances in transgenic mice overexpressing the V717F $\beta$-amyloid precursor protein. Behav. Neurosci. 5:982-990.

18. Tzavara, E.T., et al. 2003. Dysregulated hippocampal acetylcholine neurotransmission and impaired cognition in M2, M4 and M2/M4 muscarinic receptor knockout mice. Mol. Psychiatry. 8:673-679.

19. Yamamura, H., and Synder, S.H. 1972. Choline: high affinity uptake by rat brain synaptosomes. Science. 178:626-628.

20. Johnson-Wood, K., et al.1997. Amyloid precursor protein processing and Abeta 42 deposition in a transgenic mouse model of Alzheimer disease. Proc. Natl. Acad. Sci. U. S. A. 94:1550-1555.

21. Mesulam, M.M., and Mufson, E.J. 1984. Neural inputs into the nucleus basalis of the substantia inominata $(\mathrm{Ch} 4)$ in the rhesus monkey. Brain. 104:253-274.

22. Simon, J., Atweh, S., and Kuhar, M. 1976. Sodiumdependent high affinity choline uptake: a regulatory step in the synthesis of acetylcholine J. Neurochem. 26:908-922.

23. DeMattos, R.B., et al. 2001. Peripheral anti-A $\beta$ antibody alters CNS and plasma $A \beta$ clearance and decreases brain $A \beta$ burden in a mouse model of AD. Proc. Natl. Acad. Sci. U. S. A. 15:8850-8855.

24. Acquas, E., Wilson, C., and Fibiger, H.C. 1996. Conditioned and unconditioned stimuli increase frontal cortical and hippocampal acetylcholine release: effects of novelty, habituation, and fear. J. Neurosici. 16:3089-3096

25. Wang, H.-Y., et al. 2000. $\beta$-Amyloid ${ }_{1-42}$ binds to $\alpha 7$ nicotinic acetylcholine receptor with high affinity. J. Biol. Chem. 25:5626-5632.

26. Kamenetx, F., et al. 2003. APP processing and synaptic function. Neuron. 37:925-937.

27. Selkoe, D.J. 2002. Alzheimer's disease is a synaptic failure. Science. 298:789-791.

28. Cleary, J.P., et al. 2005. Natural oligomers of the amyloid- $\beta$ protein specifically disrupt cognitive function. Nat. Neurosci. 8:78-84.

29. Kotilinek, L.A., et al. 2002. Reversible memory loss in a mouse transgenic model of Alzheimer's disease. J. Neurosci. 22:6331-6335.

30. Meziane, H., et al. 1998. Memory-enhancing effects of secreted forms of the $\beta$-amyloid precursor protein in normal and amnestic mice. Proc. Natl. Acad. Sci. U. S. A. 95:12683-12688.

31. DeKosky, S.T., et al. 2002. Upregulation of choline acetyltransferase activity in hippocampus and frontal cortex of elderly subjects with mild cognitive impairment. Ann. Neurol. 51:145-155.

32. Snyder, E.M., et al. 2005. Regulation of NMDA receptor trafficking by amyloid- $\beta$ Nat. Neurosci. 8:1051-1058.

33. Slotkin, T.A., et al. 1990. Regulatory changes in presynaptic cholinergic function assessed in rapid autopsy material from patients with Alzheimer disease: implications for etiology and therapy. Proc. Natl. Acad. Sci. U. S. A. 87:2452-2455.

34. Brandon, E.P., et al. 2004. Choline transporter 1 maintains cholinergic function in choline acetyltransferase haploinsufficiency. J. Neurosci. 24:5459-5466.

35. Gates, J., Ferguson, S.M., Blakely, R.D., and Apparsundaram, S. 2004. Regulation of choline transporter surface expression and phosphorylation by protein kinase $\mathrm{C}$ and protein phosphatase $1 / 2 \mathrm{~A}$. J. Pharmacol. Exp. Therap. 310:536-545.

36. Wurtman, R.J. 1992. Choline metabolism as a basis for the selective vulnerability of cholinergic neurons. Trends Neurosci. 15:117-122.

37. Xu, J., et al. 2002. Dopamine-dependent neurotoxicity of $\alpha$-synuclein: a mechanism for selective neurodegeneration in Parkinson disease. Nat. Med. 8:600-606.

38. Nomikos, G.G., Arborelius, L., and Svensson, T.H. 1992. The novel 5-HT1A receptor antagonist (S)-UH-301 prevents (R) OH-DPAT-induced decrease in interstitial concentrations of serotonin in the rat hippocampus. Eur. J. Pharmacol. 216:373-378.

39. Bymaster, F.P., Perry, K.W., and Wong, D.T. 1985. Measurement of acetylcholine and choline in brain by HPLC with electrochemical detection. Life Sci. 237:1775-1781. 